In the case of dental radiography, as the lesions are often small, it is all the more important to pay attention to the finer details.

As regards (2) there is no question that for the best interpretation of radiographs the observer must have a good knowledge of the normal and pathological anatomy of the part. Most books dealing with the radiography of the teeth instance the interpretation of the mental and palatine foramina and loculi of the maxillary antrum as areas of periapical absorption, so that these mistakes are hardly likely to be repeated except by the beginner; even if they are, the worst that can happen is the sacrifice of one or perhaps two sound teeth.

The result of the wide knowledge of the faulty interpretation of these foramina has no doubt been responsible for the much more grave error which is brought to one's notice from time to time-that is, the interpretation of a definite periapical absorption as a normal foramen or sinus. It would almost seem that the larger the cyst the greater the possibility of it being interpreted as a shadow of the antrum. (See Fig. 8.) This is of great importance, because the systemic disease from which the patient is suffering continues or increases in severity as the suspected cause has been overlooked and allowed to remain-there being no local signs apparent to the patient or the clinical observer.

BIBLIOGRAPHY.

Hurchard and Inglis: Dental Pathology and Therapeutics. Henry Colyer, J. F. : Dental Surgery and Pathology. Longmans, Green and Co.,

Goadby, Sir Kenneth: Diseases of the Gums and Oral Mucous Membrane. Oxford Medical Press, 1928.

Hess, Walter: The Anatomy of the Root Canals of the Teeth of the Permanent Dentition. John Bale, Sons and Danielsson, 1925

Hopewell-Smith, A.: The Normal and I'athological Histology of the Mouth. $J$. and A. Churchill, 1919.

Howe, Percy R.: The Focal Theory of Infection in its Application to Teeth. National Dental A880c. Journ., vii, 1920, 635-641.

Mackey, Leonard : Dental Sepsis and Apical Infection. British Dental Journ., September 1st, 1922

Mummery, J. Howard: The Microscopic and General Anatomy of the Teeth. Oxford Medical Publications, 1924.

rice, Weston A.: Dental Infections-Oral and Systemic. The Penton Rickert. W. G.: The Status of Pulpless

1170-1178.

\section{SOME PROBLEMS OF GLYCOSURIA.*}

GEORGE J. LANGLEY, M.D.Lond., M.R.C.P., HONORARY PHYSICIAN, SALFORD ROYAL HOSPITAL.

THE introduction of insulin in the treatment of glycosuria has enabled us to overcome the urgency of diabetic coma in a way never before possible, but many problems remain.

Can the administration of insulin be regarded as a replacement therapy? There is much evidence that such is the case. It is usually recognized that the respiratory quotient rises under its influence from 0.7 , indicating metabolism of proteins and fats, to unity, indicating the metabolism of carbohydrates. The glycogen store has been shown to increase very materially under its use. The blood sugar curve-that is, the amount of sugar in the circulating, blood-is very materially altered; whereas the administration of glucose alone led in one case to a rise from 0.2 to 0.4 , the combined administration of glucose and 20 units of insulin resulted in a fall to 0.05 .

Regarding insulin, then, as an efficient replacement therapy, we are still left with very definite limitations.

1. The amount of insulin required depends upon the amount of carbohydrate to be dealt with.

2. The insulin must be given at the time of carbohydrate absorption.

3. The duration of maximum insulin efficiency would appear to be only four hours.

4. The preparation must be given hypodermically.

A consideration of these factors at once shows the very serious limitations of this line of treatment. The daily life of most persons includes four meals, which vary in themselves, and from day to day, as to their carbohydrate content. A complete replacement therapy would therefore demand four doses of insulin, given hypodermically, in ${ }^{*}$ A paper read before the Lancashire and Cheshire Branch of the quantities calculated to deal accurately with the carbohydrate content of each individual meal. This is quite impossible, and it is found that patients are very reluctant to tolerate more than two hypodermic injections daily. The diabetic patient under insulin treatment, therefore, finds himself still limited as to time, quantity, and quality of food; he must be on a diet as rigid as heretofore, although containing material previously forbidden; and, in addition, must tolerate two hypodermic punctures every day. Two of the meals will be relatively generous, while the other two are designed to contain the minimum amount of carbohydrate.

There are two schools of thought in the composition of diabetic diets. One believes that the best results are obtained by keeping the patient on the lowest diet which will maintain weight and strength, and, in consequence, the minimum possible daily dose of insulin. The other school holds that a generous diet allowing for plenty of available metabolic material, with a relatively large dose of insulin, is the better line of treatment. A careful paper by Rabinovitch in the Quarterly Journal of Medicine, in which the results of about 1,200 cases have been analysed, has led him to the conclusion that a minimal diet yields the best results. This is the type of conclusion usually arrived at by a statistical method, but it is liable to omit the human factor. It is a matter of common experience that diabetics generally not only have larger appetites in consequence of the disease, but that they have always liked or required quantities of food beyond the average. If this be so, it is possible that treatment including a generous diet will be more satisfactory to the patient and be more likely to be maintained; and it will prove much less irksome than treatment along rigidly abstemious lines. It is possible that medical practitioners generally underestimate the limitations from which these patients suffer; the continued dieting, the regularity of their meals, and the ever-recurring hypodermic punctures, with careful consideration of any food provided outside their own homes, make for a monotony which usually proves hard to bear, and the more rigid its limitations the greater the mental load. If insulin can legitimately be regarded as a replacement therapy, then, in my opinion, it should be used to replace so far as it is practicable; the introduction of double strength insulin has enabled this to be achieved more easily than was the case when only one strength was available.

The relation of proteins, fats, and carbohydrates in the total diet offers a further problem to which no generally accepted answer has yet been found. Anything approaching the usual proportions of the average meal in this country would involve the use of impossibly large doses of insulin. Even the proportion found in milk (protein 3, fat 4, carbohydrate 5) requires relatively large injections, but is probably a basis which yields good results.

Certain difficulties present themselves in relation to groups of cases. The man who has to earn his own living by hard labour obviously needs a more generous diet than the well-to-do woman with no particular occupation. The dieting of diabetic children offers great difficulty, for they must be given suitable food in sufficient quantity to permit of adequate growth, and they are nearly always serere cases. It has been my experience that no satisfactory result can be obtained among the younger patients unless three insulin doses a day are given.

Changes in dieting when the insulin dose has been established are from time to time necessary, the commonest cause, perhaps, being loss of appetite which results from some pyrexial illness. There is no doubt that this constitutes one of the greatest dangers to the diabetic to-day. If the patient does not take the carbohydrate part of his diet the insulin would appear to become unnecessary. It frequently happens that a diabetic patient contracts influenza, or some other disease associated with a rise in temperature, and is put on a low diet without insulin. The metabolic processes are increased with the rise in temperature, the carbohydrates being the chief source of heat production; in this respect the diabetic fails completely, with the result that the proteins and fats ar a inadequately split, a rapidly rising ketosis occurs, and coma becomes imminent. The occurrence of pyrexia in a 
diabetic is an indication for more insulin and more carbohydrate, not less. In my own cases I have found milk and giucose, with suifficient insulin to avoid acetone in the urine, a satisfactory line of treatment, but $I$ have always had the advantage of frequent blood sugar examinations, and have further aimed at keeping the blood sugar within reasonable limits, but at the cost of enormous doses of insulin. It is further tenable that much may be done to avoid coma by the administration by the mouth of sodium bicartonate and sodium phosphate, the action of the latter depending upon the fact that acid sodium phosphate is secreted in the urine. Large doses of both salts can be acided to each milk feed.

The experiments of Allen with partially depancreatized logs showed that plentiful carbohydrate with high blood sugar caused early death, whereas carefully regulated diets maintaining a low blood sugar allowed the dogs to live considerably longer. The question therefore arises when a diabetic with high blood sugar comes under treatment whether the maintenance of a normal blood sugar level wil! permit of any degree of pancreatic recoverr. It is $m$. experience with diabetics of middle life that if the dirt and insulin dose be correctly balanced and maintained for some months the dose of insulin required for the same diet does fall, but that the fall is slow and gradual. "No case of romplete recovery - that is to say, with a seriously. abnormal response to 50 grams of glucose-has occurred with sufficient completeness to show a normal blood sugar curve after treatment; yet a few cases have given remarkably satisfactory results, more particularly those arising from septic absorption.

The large amount of work on diabetes during the past few years has revealed a good many fallacies among our cherished beliefs. Most of us were taught that the diabetic was peculiarly liable to septic infection, whereas it is now generally recognized that septic infection is prone to givo rise to diabetes. Recently a woman, aged 32, was admitted to the Salford Royal Hospital with a carbuncle of the neck of seren days' duration. Sugar was present in the urine with much acetone, and although the patient was not in coma she was sufficiently sleepy to require rousing before she could take any notice. The slough was removed from the septic area and the blood sugar held in check by insulin. At the end of a week it was found that the patient could take a diet of over 2,000 calories, containing 85 grams of carbohydrate, without insulin and withont either glycosuria or acetonuria. She was discharged on a curtailed carbohydrate diet and without other treatment. Anothei' patient, aged 60, was known to liave had a very extensive fistula in ano and glycosuria for over ten years. Two operations and a long period of dressing produced a complete healing of the fistula, and the patient was able to take, without glycosuria, a diet restricted in carbohydrates, but permitting a very fair allowance of stout. Such cases, presumably septic in origin, occur in every diabetic clinic, and are very satisfactory. once the originating sepsis can be cleared. up.

The following case appears to me to belong to a different category. A girl, aged 18, had been treated for a fortnight for soreness of
the mouth. No local lesion was found,. and various palliatives were tried without benefit. She had previously oeen in robust health. Some degree of thirst developed; the urine was tested and was found to contain sugar. The same day she became unconscious, the urine being then loaded with sugar and acetone. The coma lasted thirty-six hours in spite of the administration of over 300 units of insulin. Recovery gradually ensued, and by the end of a week the patient was taking a diet of 1,800 calories with 40 units of insulin twice daily. Although the diet remained unchanged, the dose of insulin had to be gradually reduced on account of recurrent hypoglycaemia, - until at the end of three: weeks of treatment the diet could be taken without insulin, and without. either glyt the die acetonuria. At the end of a month's treatment she glycosuria o on the same diet but without insulin and remained returned home well with no glycosuria. Six weeks later the festivities of Christmas proved too much for her self-control, and after two days of feasting the urine was loaded with sugar, which did not disappear until the original diet of 1,800 calories had been regularly adhered to for a week. Since then there has been no lapse, and the patient remains very well.

The temptation is almost irresistible to belieio that diabetes in voung subjects has an acute and infective origin; the onset in many of these cases suggests it si strongly. If this be so, and they are adequately treatcd from the first, to avoid any long period of raised blood sugar, which is known to be so deleterious to the islet tissue, it is surely not too much to hope that some of them will actually recorcr completels, and so be saved from the disease.

The feasibiity of operation upon diabetic patients has uniergone complete change with the introduction of insulin. 'ine visual method adepted is to give the : patient an adequate diet and the required insulin dose to maintain frcedom from glycosuria for a week. On the day of operaticn the usna? purging in preparation is dispensed with, and the patient before receiving the anaesthetic is given 50 grams of giucoss anc? 10 to 20 units of insulin, dependent upon the amount of insulin required for his diet, as forming an estimate of the severity of his pancreatic deficiency. Chloroform is countermanded as an anaesthetic, but ether appears to be quite safe. Post-anaesthetic vomiting and the general dietary upset, consequent upon the anaesthetic, may occasion-some difficulty in determining the required insulin close for the first two or three dars after the operation, otberwise this procedure causes little tioubic.

Some time ago a woman, aged 38 , who had been known to have diabetes for ten years, went into coma and remained unconscious for six days, in spite of very large insulin doses. She ultimately made a very good recovery, and led a fairly useful life, taking made a very good recovery, and led a fairly useful life, taking about 100 units of insulin daily; she was regarded as a severe case
of diabetes. A year later she was seized with severe abdominal pain and vomiting, añd some hours later a gangrenous appendix was surcessfuliy removed by Mr. Macalpine. The patient took the anaesthetic weli, and made a remarkably good recovery without ans signs of coma. The wound healed up well, as in ordinary cases. She unfortunately developed femoral thrombosis, which required prolonged rest in bed, but she is still alive and well and able to lead a normal life except for hnr dietary restrictions.

To vidertake a laparotomy in this particular case appeaied to be courting disaster, but very little trouble was experienced from her diabetic condition, and I persona jo not regard diabetes as a contraindication $t$ o operation to-day.

It is now generaily recognizer that glycosuria does not always man diabetes, and renal glycosuria in which the kidney passes sugar with the urine at an abnormally low concentration in the blood is a fairly common occurrence. But there are three other conditions which are perhaps not quite so frequently looked for and understood. The occurrence of sugar in the urine after mental excitement is fairly common. It was found in a considerable percentage of students during final examinations, and in football teans after exciting and important matches, and is believed to be due to the physiological mobilization of sugar resulting from increased adrenal secretion. During the past year three cases have come under $\mathrm{mr}$ notice in which the diagnosis between cerebral haemorrhage and diabetic coma had to be made. All of them were very extensive cerebral hacmorrhages, but all the patients had glycosuria, presumably caused by the haemorrhage, as one of them was known not to have had any urinary sugar on the morning of the stroke. The third condition is the frequent association of diabetes with exophthalmic goitre. In the majority of hyperthyroid cases the tolerance for sugar is within the normal limits, while in others there is a very definite intolerance, with abnormal rise and slow fall in the blood sugar content. These cases appear to be materially improved by insulin treatment, both as regards their diabetes and their hyperthyroidism.

Lately I have had under my care a patient with wellmarked hyperthroidism, who has a very low renal leak point and slight carbohydrate intolerance. In spite of this, three-quarters of the thyroid was successfully removed by Mr. Jefferson without any untoward event and with considerable bencfit, but without the help of insulin.

Some form of therapy which can be administered by the mouth, and with a longer period of activity than that of insulin, is still being diligently sought. - No such thing has as yet been found, but two attempts have been made. Insulin can be absorbed in the stomach, but only in very small quantities, if given either: with alcohol or with saponin, but the quantity is not sufficient to make it practicable. The other attonpt depends upon the fact that guanidine was thought by Noël Paton and Findlay to have a definite effect in-lowering the hlood sugar. The drug is much too toxic to he emplcyed, but various derivatives have 
been tried, and some can be tolcrated. A product of this group known as sinthalin is now on the market. It is doubtful whether it can be administered in sufficient dases to control the blood sugar, but there is no doubt that the drug does give rise to considerable gastritis and some diarrhoea. This is likely to control the amount of food ingested, and by so doing will materially improve the middle-aged diabetic, who eats and drinks too much.

\section{PULMONARY FIBROSIS :}

An Investigation into the Obigin and Cockse. BY

C. ve W. KITCAT, M.R.C.S., L.R.C.P., RESIDENT MEDICAL OYFICER, BROMPTON HOSPITAL FOR DISEAEES OF THE CHEST; $\triangle N D$

T. HOLMES SFLLORS, M.A., B.M.Oxon., CaSUALTY SCRGiCAl OFFICER, MIDDLEEEX HOSPITAL; G. H. HUNT TRAVELLING SCHOLAR, UNIVERSITY OF OXFORD.

Pulmonary fibrosis in childhood inflicts such a physical handicap on its victims in later life that a short account of our attempts to investigate the origin and subsequent course of the disease may be of general interest and lead to further study. The materials for the investigation have been gathered from the records of the Brompton Hospital, and in the main confirm those recently published by $\mathrm{Dr}$. Burton Wood." We are using the term "pulmonary fibrosis" because it is at present the most familiar designation of the disease known as fibrosis, fibroid induration, chronic broncho-pneumonia, chronic basal pulmonary catarrh, and early bronchiectasis-which titles, we believe, indicate phases of the same condition.

Pulmonary fibrosis may be a disease entity per se, but it is commonly secondary to an attack of bronchopmeumonia, usually of the subacute variety, following some of the infectious fevers, especially measles and whoopingcough. Broncho-pneumonia invariably produces some degree of bronchiolar dilatation, and persistence of the inflammation promotes fibroid thickening of the bronchioles and air cells; moreover, the slight dilatation is in itself a cause of the cough which tends in vicious sequence to increase the weakness of the tubular walls. Pulmonary fibrosis begins in early childhood, a fact explained partly by the special liability at that age to infection by measles and whooping-cough, and partly by certain peculiarities in the anatorny of the lungs-namely, the relatively large calibre of the bronchi, the thickness of their epithelial lining, and the thick, but yielding, walls of the alveoli; these are embryonic and infantile distinctions which have disappeared in a healthy child by the fifth year.

Quite early in the investigation we found it essential to impose certain arbitrary limits to our search so as to keep it within reasonable bounds, and therefore made use of only such cases as fulfilled the following conditions:

(a) The age at the first examination must have been under 15 years.

(b) Each case selected must have been under observation for at least six months.

(c) Physical signs must have been present at the first examination and persisted over the whole period of attendance.

(d) At least five years must have elapsed since the last attendance.

Cases with any evidence of pulmonary tuberculosis or established bronchiectasis (at the first attendance) were excluded.

More than 23,000 notes from the out-patient department of the Brompton Hospital were reviewed, and 259 patients apparently suitable for investigation were followed up. Of these, 7 were found to have died and are not included in this report, but 53 attended and form the basis of inquiry.

At our examination a full clinical survey was made, with special reference to the following features: (a) Clinical history since last attendance at hospital. (b) Symptoms with particular reference to normal life and work. (c) Physique and capacity for work. (d) Physical signs in lungs.
Cissification.

The 53 cases are classified from an economic standpoint; we use the term "fit" to imply the capacity of an individual for a normal useful life.

1. Fit, without signs or symptoms-resolution has taken place in the interval

2. Fit, but with physical signs-permanent injury to lung tissue, but no apparent impairnent of general health

3. Not fit and with physical signs-permanent injury to lung tissue accompanied by some degree of constitutional disability ...

4. Bronchicetasis has supervened since last examined

\section{Comments.}

Complete Resolution.-This group is small but definite; tho notes show that no improvement had accrued during the period of treatment, and it is impossible to judge the ago at which the induration resolved.

lersistent Signs, but no Symptoms. - It is remarkable that a good physique and geweral health can be maintained in spite of permanent damage to lung tissue.

Persistent Fibrosis. - These a ases are the most numerous, and, with the next group, eonstitute a grave indictment of present treatment of the disease. The srmptoms and signs are compatible in most cases; there is an increased tendency to coughs and colds, and the majority are confined to bed or house at frequent intervals during the winter months.

Bronchiertasis.--As mentioned previousls, we rejected cases which showed the presence of established bronchiectasis while attending hospital, and this group only includes those in which the condition has developed since last seen. The number is small, but in addition the accomts of the seren deatins suggested this as the terminal cause.

syphilis.-None of the patients examined exhibited clinical evidence of svphilis.

Pulmonary Tuberculosis.-All patients were cxamined for tuberculosis, and oniy one was found to have tubercle bacilli in the sputum. This case is not included in our figures.

$$
\text { Cacisation. }
$$

The primary causes were:

\begin{tabular}{|c|c|c|c|}
\hline & & & Cases. \\
\hline Measles alone & ... & ... & $14(26.5 \%)$ \\
\hline Whooping-cough alone & ... & $\ldots$ & $8(15 \%)$ \\
\hline Measles with whooping-cough & $\ldots$ & $\ldots$ & $16(30 \%)$ \\
\hline "Pncumonia $" \quad \ldots \quad$... & $\ldots$ & & $14(26.5 \%)$ \\
\hline Scarlet Fever $\quad \ldots$ & $\ldots$ & $\ldots$ & $1(2 \%)$ \\
\hline
\end{tabular}

An accurate history of broncho-pneumonia following these ferers could not be definitely established, but was suggested in the majority of cases. Broncho-pnenmonia is a frequent and often fatal complication of measles, and among the poor or weakly is apt to run an irregular but persistent course. It may last as long as six weeks, or even more, and in such a case fibrosis will probably ensue. Bronchopneumonia is also the most serious complication of whooping-cough, and accounts for nine-tenths of the deaths from the disease. Our cases support the view that these two infections are mainly responsible for the production of pulmonary fibrosis. As the severity of both decreases with each year after the second, the fatalistic attitude of the laity - "let them catch it and get it over"-is to be deplored; since after the fifth year there is little danger of severe pulmonary complications to be feared from the infection or its sequel, every effort should be engaged to guard young children from any risk of contracting either, and especially should children with measles be protected from whooping-cough. "Pneumonia" appears frequently in the histories and gives rise to some confusion, for lobar inflammation is probably not intended; it is not a frequent complication of the fevers, and if present is always of a lobular variety. It would appear likely that a combination of impaired percussion note, weakened puerile breath sounds, and crepitations occurring in a febrile child might well at first sight suggest a diagnosis of lobar pneumonia, whereas actually the condition represents an exacerbation in a fibrotic lung. 